\title{
Density-driven convection enhanced by an inclined boundary: Implications for geological $\mathrm{CO}_{2}$ storage
}

\author{
Peichun A. Tsai, ${ }^{1,2, *}$ Kathleen Riesing, ${ }^{1}$ and Howard A. Stone ${ }^{1}$ \\ ${ }^{1}$ Department of Mechanical and Aerospace Engineering, Princeton University, Princeton, New Jersey 08544, USA \\ ${ }^{2}$ Group of Soft-matter, Fluidics and Interfaces, MESA + Institute for Nanotechnology, Faculty of Science and Technology, \\ University of Twente, 7500 AE Enschede, The Netherlands
}

(Received 22 April 2012; published 31 January 2013)

\begin{abstract}
We experimentally examine dissolution-generated, density-driven convection with an inclined boundary in both a Hele-Shaw cell and in a porous medium. The convection, manifested by descending, dense fingers, is generated by a diffusive mixing of two liquids at the interface. We investigate the dynamics, widths, and wavelengths of the fingers and characterize the global convective transport for a wide range of permeabilities and tilt angles of the boundaries. Our results have implications for $\mathrm{CO}_{2}$ storage in a saline aquifer when brine saturated with $\mathrm{CO}_{2}$ produces a heavier mixture, which may result in an enhanced mass transfer by convection. Our measurements reveal a further enhancement of convection with inclined boundaries, which suggests that sloping formations provide improved sites for $\mathrm{CO}_{2}$ storage.
\end{abstract}

DOI: 10.1103/PhysRevE.87.011003

PACS number(s): 47.20.Bp, 47.15.gp, 47.56.+r

Convection due to a density difference in fluids is ubiquitous and, moreover, impacts energy and mass transfer in nature and industry. For instance, continental drift due to mantle convection, climate conditions in the atmosphere, and largescale circulations of the ocean currents can be attributed to density-driven motions [1]. A variety of technological applications utilize convective processes to enhance chemical reaction rates, sedimentation speeds, and heat transport [1-4]. Finally, the technology of carbon capture and sequestration (CCS) recently proposed for greenhouse gas mitigation relies on dissolution-generated convection to secure the long-term storage of $\mathrm{CO}_{2}$ in deep saline formations [5-7].

The CCS technology consists of transporting $\mathrm{CO}_{2}$ from a source (such as power plants), purifying, pressurizing, and then storing it in the subsurface (about 1-2 km underground). Among various potential storage sites, natural deep saline formations, which are porous media saturated with brine, present a significant capacity [5]. At the reservoir conditions, $\mathrm{CO}_{2}$ is thermodynamically supercritical, with a liquidlike density $\left(500-800 \mathrm{~kg} / \mathrm{m}^{3}\right)$ less dense than saline $\left(\approx 1000 \mathrm{~kg} / \mathrm{m}^{3}\right)[5,8]$. Thus the $\mathrm{CO}_{2}$ is buoyant and slowly rises through porous rocks until it is confined by impermeable (cap) rocks above the saline layer. Via diffusion, $\mathrm{CO}_{2}$ gradually dissolves into saline, such that the mixture is heavier than either fluid by approximately $1 \%$ [8]. This dense interfacial layer is gravitationally unstable and convection may set in. The convection, in contrast to the slow diffusive dissolution, significantly enhances the downward transport of $\mathrm{CO}_{2}$ and upward motions of fresh brine. This convective process is important for the long-term storage of $\mathrm{CO}_{2}$ because the chemical mineralization of porous rocks due to $\mathrm{CO}_{2}$ dissolution will eventually take place [5].

Motivated by convection in the context of CCS, several studies of the dissolution-driven convection have been recently undertaken using theory $[9,10]$, simulations [11], and experiments [12-15]. The onset of convection and the dissolution rates for different permeabilities in model systems and in porous media have been reported. Most studies are carried out

*Corresponding author: p.a.tsai@utwente.nl in vertical cells, but some geological formations are naturally inclined. For example, the Hontomin pilot storage site in Spain has a domelike formation with a slope close to $20^{\circ}$ where $\mathrm{CO}_{2}$ will be injected at a depth about $1450 \mathrm{~m}$ [16]. Because of the difficulty of producing subsurface conditions of temperature and pressure, there are few experimental studies under similar conditions [17]. In this study, we carry out experiments [12,14] and report the measurements of the dissolution rates for a wide range of permeabilities. In addition, motivated by naturally inclined geological formations [18], we perform experiments with sloping boundaries to investigate the possibility of enhanced convection due to density-driven motions with inclined boundaries.

The experimental apparatus (see Fig. 1) consists of a Hele-Shaw cell of two parallel plates uniformly separated by a narrow space. The gap is controlled by a thin stainless-steel shim of thickness $b$, which is sandwiched by two transparent polycarbonate sheets of thickness $1.3 \mathrm{~cm}$. The Hele-Shaw configuration provides a useful model for studying porous media flow because the Darcy description holds with the permeability $k=b^{2} / 12$ for small Reynolds number [20]. The size of the cell is $10.2 \times 10.2 \mathrm{~cm}^{2}$, and different shim thicknesses $b$ are used to examine the effect of permeability. In addition, glass beads of different diameters $d$ (between $750 \mu \mathrm{m}$ and $1.3 \mathrm{~mm}$ ) packed to a depth $\approx 5 \mathrm{~mm}$ are used. The permeability for the packed beads is estimated using the Kozeny-Carman model [20] with the average $d$ and porosity $\phi \approx 0.37$. Therefore, the explored range of $k$ for the packed beads is between $3.3 \times 10^{-5}$ and $2.15 \times 10^{-4} \mathrm{~cm}^{2}$. In addition, the vertical cells are rotated at an angle $\alpha$ counterclockwise to investigate the influence of inclined boundaries.

We use water and propylene glycol (PPG) to replace $\mathrm{CO}_{2}$ and brine, respectively, to produce approximately a $1 \%$ increase in the density of the mixture [14]. We initially inject denser propylene glycol $\left(\rho_{\mathrm{PPG}}=1.035 \mathrm{~g} / \mathrm{cc}\right)$ into the bottom of the cell (to a vertical height on average about $5 \mathrm{~cm}$ ) and then slowly inject water $\left(\rho_{\mathrm{H}_{2} \mathrm{O}}=1.000 \mathrm{~g} / \mathrm{cc}\right)$ into the top of the cell. The liquid stratification is initially stable; however, at the interface the mixture of water and PPG creates a denser interfacial layer for water concentrations less than $50 \%$. In 
(a)

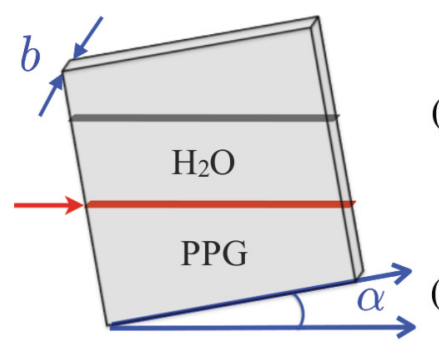

(b)

(c)

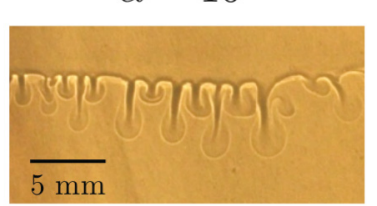

(d)
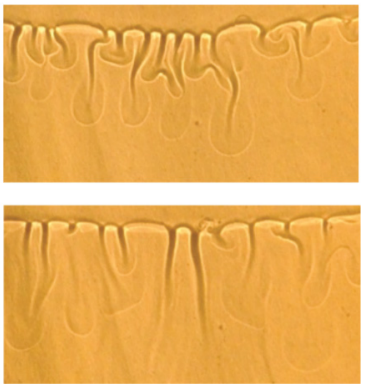

FIG. 1. (Color online) (a) Schematic diagram of the experimental setup of a vertically placed Hele-Shaw cell, two parallel plates with a uniform and small separation $b$, at a tilt angle $\alpha$. (b)-(d) Shadowgraph snapshots of the descending plumes due to the dense mixture at the interface [marked by the horizontal red arrow in (a)] between water and propylene glycol (PPG, heavier than water) in a Hele-Shaw cell of a permeability $k=b^{2} / 12=2.2 \times 10^{-4} \mathrm{~cm}^{2}$ and $\alpha=(10 \pm 0.3)^{\circ}$. The corresponding time sequences are (b) 270, (c) 555, and (d) $1125 \mathrm{sec}[19]$.

general, the exact density of the mixture depends on the composition [14]. Here we used the same liquids as in Ref. [14] and thus the same liquid parameters. The temperature of the fluid cell is in equilibrium with the room temperature of the laboratory, which acts as a thermal bath, at $23 \pm 0.2{ }^{\circ} \mathrm{C}$.

The dense interfacial layer due to dissolution may exhibit a gravitational instability when the diffusion layer is thick enough [9]. Here we only focus on the dynamics beyond the onset of the convection. Figures 1(b)-1(d) illustrate the evolution of the dense plumes. The density gradient sharpens the $\mathrm{H}_{2} \mathrm{O}$-PPG interface, which is clearly revealed in the shadowgraph images. The time evolution of the interface provides a direct measure of the dissolution rate, thereby the convective flux [12]. As the dense plumes advect downward, more fresh surrounding fluid is brought to the interface. In the experiments, the $\mathrm{H}_{2} \mathrm{O}-\mathrm{PPG}$ interface moves upward at a constant speed, $V_{I}$, for a long time $(\approx 100$ mins $)$ and finally stops when the lower liquid reservoir becomes denser due to dissolution. In other words, the dissolution eventually decelerates for a $\mathrm{CO}_{2}$-rich brine reservoir.

Here we characterize the maximum convective flux by measuring the constant speed of the moving interface, $V_{I}$. From the captured snapshots [as shown in Figs. 1(b)-1(d)], we use IMAGEJ software to calculate $V_{I}$ by performing linear fits of the displacement-time data of the interface. In detail, for each experiment, the time evolutions of ten randomly chosen interfacial locations are measured to obtain $V_{I}$. The dimensionless forcing term is characterized by the DarcyRayleigh number $\mathrm{Ra}$, which is similar to the Rayleigh number in thermal convection: $\mathrm{Ra}=U H / \phi D$ [21], where $H$ is the depth of the reservoir, $\phi$ is the porosity, $D$ is the diffusivity, and $U=k g \Delta \rho / \mu$ is the Darcy buoyancy velocity based on the permeability $k$, maximum density difference $\Delta \rho$, gravitational

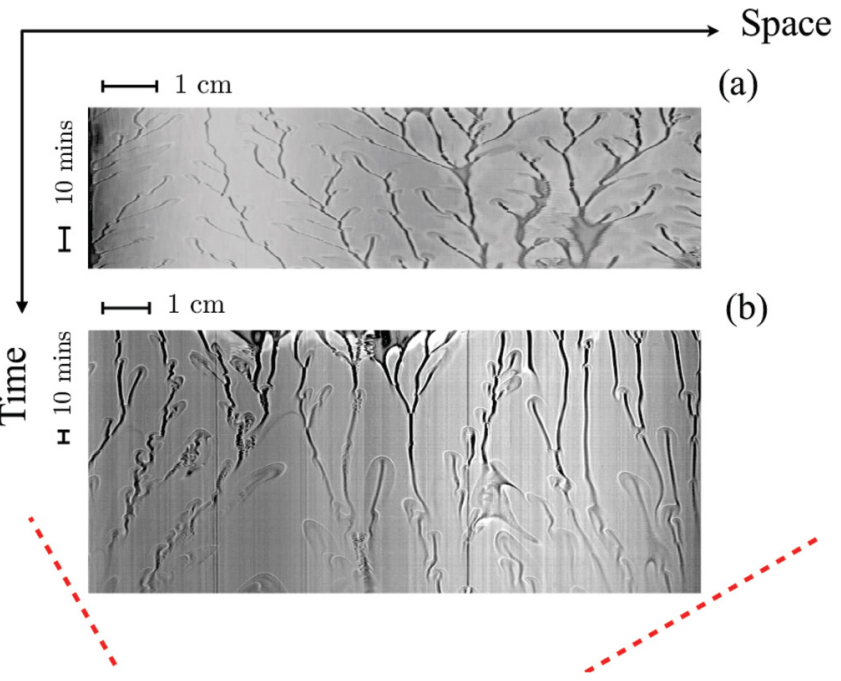

FIG. 2. (Color online) Representative spatiotemporal diagrams of the dense fingers (shown by the darker intensity) close to the interface where the dissolution takes place in a vertical cell of $\alpha=0^{\circ}$ in (a) and in an inclined cell of $\alpha=30^{\circ}$ in (b). As revealed in (b), the plumes move laterally towards the inclined walls at a later time. The two dashed lines below (b) schematically represent the slopes of the inclined boundaries.

acceleration $g$, and the viscosity $\mu$ [14]. The dimensionless convective flux, characterized by the Nusselt or the Sherwood number, is the ratio of total mass flux to the diffusive counterpart: $\mathrm{Nu}=F_{c} /(\phi \Delta D / H)$, where the convective flux $F_{c}=\phi V_{I} \Delta$ is based on the concentration difference between the liquids, $\Delta$ [12], and thus $\mathrm{Nu}$ can be directly measured via $\mathrm{Nu}=V_{I} H / D$.

The convective flux occurs in the form of descending plumes. Their dynamics is displayed in Fig. 2, revealing a spatiotemporal diagram of the conduits of the traveling plumes close to the interface. Phenomenologically, plumes move downward due to gravity and gradually merge, as revealed in Fig. 2(a) for a vertical cell. Similarly, with inclined boundaries, plumes advect downward and merge, as shown by the coarsening patterns in Fig. 2(b). In addition, plumes adjacent to the interface later in time move laterally toward the inclined boundaries, which enhances the large-scale convection.

Next, we quantitatively analyze the plume statistics for different permeabilities and tilt angles (Fig. 3). In particular, the plume width $\delta$ is measured using IMAGEJ software with the shadowgraph snapshots of the plume (see the inset in Fig. 3 as an example) and for the Hele-Shaw cells we find that $\delta$ is constant, $\delta=0.080 \pm 0.005 \mathrm{~cm}$, for the same $k=2.2 \times$ $10^{-4} \mathrm{~cm}^{2}$ for different tilt angles: $\alpha=0^{\circ}, 10^{\circ}$, and $20^{\circ}$. Physically, $\delta$ is determined by the gap of the Hele-Shaw cell (or $k$ ) [9] and thus is not affected by the inclined boundaries for the same $k$. In addition, the results in Fig. 3 reveal an insignificant influence of the tilt angle on the plume statistics in terms of the dimensionless ratio of the plume width to the average wavelength, $\delta /\langle\lambda\rangle$. The general trend of $\delta /\langle\lambda\rangle$ in time consists of a rapid decay, reflecting the coarsening of the plumes, and a saturation due to eventually infrequent generation of the plumes. The results for the initial values of $\delta /\langle\lambda\rangle$ agree well with that of a linear stability analysis at the onset of convection, 


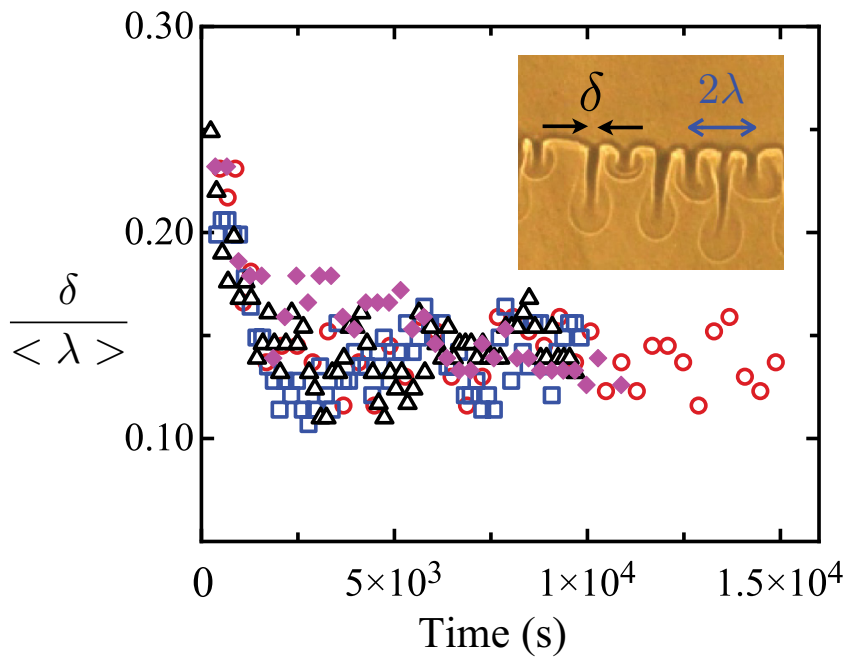

FIG. 3. (Color online) Time evolution of the plume statistics in a Hele-Shaw cell in terms of the ratio of the plume width $\delta$ to the average wavelength $\langle\lambda\rangle$ for different parameters in $(k, \alpha): \alpha=0^{\circ}(\circ), \alpha=10^{\circ}$ $(\square)$, and $\alpha=20^{\circ}(\triangle)$ for the same permeability $k=2.2 \times 10^{-4} \mathrm{~cm}^{2}$, and $\alpha=0^{\circ}, k=1.3 \times 10^{-4} \mathrm{~cm}^{2}(\diamond)$. The inset is a representative snapshot of the descending plumes, showing the measured width $(\delta)$ and wavelength $(\lambda)$ of the plumes.

which predicts $\delta /\langle\lambda\rangle=0.27$ [9]. With a different permeability $k=1.3 \times 10^{-4} \mathrm{~cm}^{2}$ the data of $\delta /\langle\lambda\rangle$ follows a similar trend, but with a slight decrease of the average plume wavelength. In short, the inclined boundaries (of the same $k$ ) do not alter the initial convective instability, but the permeability does.

In the context of $\mathrm{CCS}$, the dissolution rate of $\mathrm{CO}_{2}$ into surrounding brine in a porous medium is characterized in dimensionless terms by the Nusselt number, Nu. It is crucial to identify suitable geological saline formations, given the permeability, dimensions, and characters of the reservoir (e.g., in terms of $\mathrm{Ra}, \alpha$, etc.), by estimating $\mathrm{Nu}$. We experimentally examine the Nu-Ra relation by changing $k$ for $\alpha=0$ using both Hele-Shaw cells and porous media of packed beads. We found that in the parameter range of $10^{4} \lesssim \mathrm{Ra} \lesssim 10^{5}$, a scaling law $\mathrm{Nu}=A \mathrm{Ra}^{\beta}$ exists, with the best fit of $A=$ 0.037 , and $\beta=0.84$ in our experiments (see Fig. 4). This power law agrees well with existing experimental results of $\beta \approx 4 / 5$, found in porous media of beads [12] and in similar Hele-Shaw cells with different reservoir dimensions [14]. In contrast to Rayleigh-Bernard thermal convection where several theoretical, experimental, and numerical studies have been performed [1], the physical origin of the density-driven $\mathrm{Nu}$-Ra scaling in porous media is still unclear. This advectionassisted dissolution grows less steeply with $\mathrm{Ra}$ than the situation of thermal convection in porous media $\mathrm{Nu} \sim \mathrm{Ra}$, which is normally expected [12]. The $\mathrm{Nu} \sim \mathrm{Ra}^{4 / 5}$ scaling has been suggested by a theoretical argument in which the lateral compositional diffusion between dense downward and buoyant upward plumes decrease the concentration and thus density difference, thereby decreasing the power law away from the linear scaling of $\mathrm{Nu}$ with $\mathrm{Ra}$ [12]. For the CCS applications and for various potential geological sites, using the obtained Nu-Ra relation we can estimate the relevant time scale of faster dissolution due to convection than that of the slow process due to pure diffusion. For instance, the operating

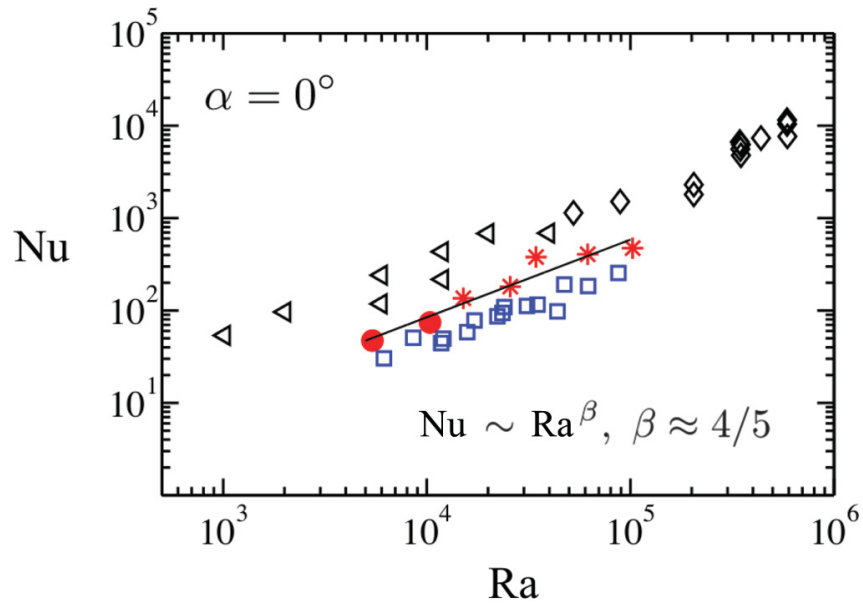

FIG. 4. (Color online) The effect of permeability on the dimensionless convective mass transfer in vertical cells $\left(\alpha=0^{\circ}\right)$. The Darcy-Rayleigh number, $\mathrm{Ra}$, is varied using Hele-Shaw cells with different gaps $(*)$ and using porous media of beads $(\bullet)$. The solid line shows the best power-law fit of our data with the scaling exponent 0.84 . The upper numerical $(\triangleleft)$ and experimental $(\diamond)$ data are from Fig. 3 of Ref. [12] with different liquids and the lower data ( $\square$ ) are obtained from Ref. [14] for vertical Hele-Shaw cells of different aspect ratios.

Sleipner site in the North Sea has been reported with an estimated $\mathrm{Ra} \approx 1.2 \times 10^{4}$ [22], and our experimental finding of $\mathrm{Nu} \approx 100$ predicts that the time to convectively mix $\mathrm{CO}_{2}$ is shortened to a time scale of $\approx 63$ years, from the diffusive mixing time $t_{d} \sim H^{2} / D \approx 6300$ years.

In addition, the results in Fig. 4 indicate different prefactors in the Nu-Ra scalings found in various experiments in spite of the similar exponent $\approx 4 / 5$. This feature may imply that the $\mathrm{Nu}$-Ra power law may be affected by the details of the experimental situations, such as the dependence on the aspect ratio of the cells and liquid properties. In the classical thermal or electrical convection, varying the Prandtl number and the aspect ratio of the convection cell influences the Nu-Ra scaling [23-27]. Here, similar mechanisms may affect the $\mathrm{Nu}$-Ra scaling, via either the exponent or the prefactor; these investigations are intriguing but beyond the scope of the Rapid Communication.

To examine the influence of the inclined boundaries, we measure and compare the speed of the fluid-fluid interface $V_{I}$. The inclined cells have a varying cell height $H$, and thus the dimensionless $\mathrm{Nu}$ and $\mathrm{Ra}$ numbers are not uniquely determined. In addition, to eliminate the enhanced transport due to the naturally increasing interfacial contact area for an inclined cell, we report in Fig. 5 the normalized dissolution speed of an inclined Hele-Shaw cell by that of a vertical one (speed $V_{0}$ ) of the same permeability. The error bars present the scatter of the results from at least three or four independent experiments. We observe that the inclined boundaries increase the propagation speed by about $20 \%$ on average compared to the counterpart of a vertical Hele-Shaw cell with $\alpha=0^{\circ}$. The data also show a steady increase in $V_{I} / V_{0}$ for the range of tilt angle we have studied.

We suggest a mechanism for this enhancement analogous to the Boycott effect, where the sedimentation speed of heavy, suspended particles is significantly increased in a tilted tube [2]. Phenomenologically, the dense plumes in porous 


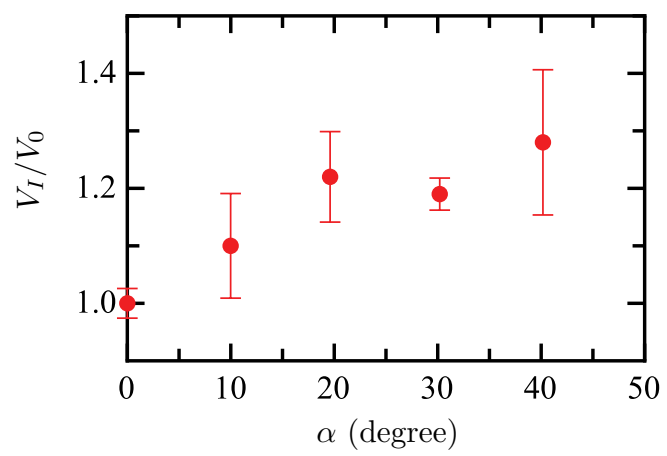

FIG. 5. (Color online) Density-driven Hele-Shaw convection with inclined boundaries reveal a larger speed of the interfacial propagation, $V_{I}$, in comparison to that in a vertical cell, $V_{0}$, with a fixed permeability $k=2.2 \times 10^{-4} \mathrm{~cm}^{2}$ [28].

media of packed beads move vertically first and subsequently move along the inclined boundaries due to the component of gravitational acceleration projected along the boundaries (see the supplementary movies for Fig. 5). The coherent movement of plumes along the inclined boundaries creates an easier pathway for dense plumes. Then the plumes close to the liquid interface have lateral motions towards the side of the cell. The resulting plume movements appear to set up large-scale sedimentation currents, qualitatively similar to the Boycott case, where bulk convection enhances the particle sedimentation [3].
Theoretical analysis for the convection requires further work, and a modified calculation of the Boycott situation will be insightful. For useful CCS applications, our experimental findings suggest that for a given permeability an improved choice of a geological site should have a sloping formation.

In conclusion, a Nu-Ra scaling for density-driven convection due to a dissolution in porous media is verified experimentally; the $\mathrm{Nu} \sim \mathrm{Ra}^{4 / 5}$ scaling was observed in our experiments for $10^{4} \lesssim \mathrm{Ra} \lesssim 10^{5}$, showing that the dissolution will be increased by $85 \times$ to $585 \times$, respectively, due to the advective process compared to the purely diffusive counterpart. Slightly beyond the onset of convection, the plume width and average wavelength are not altered by the inclined boundaries, for the same permeability. However, subsequently lateral and coherent movements of dense plumes adjacent to the liquid-liquid interface and along the inclined boundaries are observed in an inclined cell. In addition, the speed of the interfacial propagation is enhanced with inclined boundaries, which suggests that for a suitable permeability it is beneficial to choose $\mathrm{CO}_{2}$ storage sites with sloping geological formations.

The authors thank T. Al-Housseiny, M. Celia, H. E. Huppert, J. Nordbotten, R. Socolow, and Z. Zheng for stimulating discussions. We thank BP and the Carbon Mitigation Initiative at Princeton University for partial support of this research. P. Tsai thankfully acknowledges the support of Taiwan NSC through Grant No. NSC98-2917-I-564-138.
[1] G. Ahlers, S. Grossmann, and D. Lohse, Rev. Mod. Phys. 81, 503 (2009).

[2] A. Boycott, Nature (London) 104, 532 (1920).

[3] A. Acrivos and E. Herbolzheimer, J. Fluid Mech. 92, 435 (1979).

[4] T. Seon, J. P. Hulin, D. Salin, B. Perrin, and E. J. Hinch, Phys. Fluids 17, 031702 (2005).

[5] E. Rubin and H. de Coninck, IPCC Special Report on Carbon Dioxide Capture and Storage (Cambridge University Press, New York, 2005).

[6] S. Anderson and R. Newell, Annu. Rev. Environ. Resour. 29, 109 (2004).

[7] S. Bachu, Prog. Energy Combust. Sci. 34, 254 (2008).

[8] S. Bachu and J. J. Adams, Energy Convers. Manage. 44, 3151 (2003).

[9] A. Riaz, M. Hesse, H. A. Tchelepi, and J. F. M. Orr, J. Fluid Mech. 548, 87 (2006).

[10] M. Javaheri, J. Abedi, and H. Hassanzadeh, Transp. Porous Med. 84, 441 (2010).

[11] H. Hassanzadeh, M. Pooladi-Darvish, and D. W. Keith, AIChE 53, 1121 (2007).

[12] J. A. Neufeld, M. A. Hesse, A. Riaz, M. A. Hallworth, H. A. Tchelepi, and H. E. Huppert, Geophys. Res. Lett. 37, L22404 (2010).

[13] T. J. Kneafsey and K. Pruess, Transp. Porous Med. 82, 123 (2010).

[14] S. Backhaus, K. Turitsyn, and R. E. Ecke, Phys. Rev. Lett. 106, 104501 (2011).

[15] A. C. Slim and T. S. Ramakrishnan, Phys. Fluids Phys. Fluids 22, 124103 (2010).
[16] V. Vilarrasa, S. Olivella, and J. Carrera, 3rd Workshop of CODEBRIGHT Users (UPC, CIMNE, Spain, Barcelona, 2011), p. 1.

[17] T. Suekane, S. Soukawa, S. Iwatani, S. Tsushima, and S. Hirai, Energy 30, 2370 (2005).

[18] D. Vella and H. E. Huppert, J. Fluid Mech. 555, 353 (2006).

[19] See Supplemental Material at http://link.aps.org/supplemental/ 10.1103/PhysRevE.87.011003 for a representative shadowgraphic movie of the dissolution-driven convection with an inclined boundary.

[20] J. Bear, Dynamics of Fluids in Porous Media (Elsevier, New York, 1972).

[21] J. Ennis-King, I. Preston, and L. Paterson, Phys. Fluids 17, 084107 (2005).

[22] M. Bickle, A. Chadwick, H. E. Huppert, M. Hallworth, and S. Lyle, Earth Planet. Sci. Lett. 255, 164 (2007).

[23] Z. A. Daya and R. E. Ecke, Phys. Rev. E 66, 045301(R) (2002).

[24] P. Tsai, Z. A. Daya, and S. W. Morris, Phys. Rev. Lett. 92, 084503 (2004).

[25] P. Tsai, Z. A. Daya, and S. W. Morris, Phys. Rev. E 72, 046311 (2005).

[26] H.-D. Xi and K.-Q. Xia, Phys. Rev. E 78, 036326 (2008).

[27] J. Q. Zhong, R. J. A. M. Stevens, H. J. H. Clercx, R. Verzicco, D. Lohse, and G. Ahlers, Phys. Rev. Lett. 102, 044502 (2009).

[28] See Supplemental Material at http://link.aps.org/supplemental/ 10.1103/PhysRevE.87.011003 for a comparison of two movies of the dissolution-driven convection in porous media, one with and the other without inclined boundaries. 